\title{
Synthesis and optimization of Novel Chitosan/Cellulose Acetate Natural Polymer Membrane for water treatment
}

\author{
M. Kamal ${ }^{1}$, E.M. Abdelrazek ${ }^{1,2}$, N.M. Sellow ${ }^{1}$, A.M. Abdelghany ${ }^{3}$ \\ 1Physics Department, Faculty of Science, Mansoura University, Mansoura, 35516, Egypt. \\ ${ }^{2}$ Physics Department, Faculty of Science, Al-Ula, Taibah University, Madina, Saudi Arabia. \\ ${ }^{3}$ Spectroscopy Department, Physics Division, National Research Center, 33 Elbehouth St., Dokki, 12311, Giza, \\ Egypt.
}

\begin{abstract}
Chitosan (Ch) and Cellulose Acetate (CA) natural polymer in addition to other samples with different mass fractions of Chitosan/Cellulose Acetate (Ch/CA) were synthesized via simple casting route. Prepared samples were studied using FT-IR and UV/vis spectroscopic techniques. Structural variations due to the process of blending were retraced using X-ray diffraction data (XRD) and morphological investigation using scanning electron microscopy (SEM). Obtained spectral data reveals compatibility and complexation between polymer constituents identified through presence of polymer characteristic peaks with small variation in both intensity and position along with change in the value of optical energy gap from UV optical absorption spectral data. XRD pattern indicate the semicrystalline nature of the studied sample with an observable change in the crystallinity. Moreover, scanning electron microscopic (SEM) micrographs reveals homogenous structure without any evidence for agglomerations nearly in all compositions.
\end{abstract}

\section{Keywords:}

Polymer Blend; SEM; Natural polymer; FT-IR

Corresponding Author

E-mail: nm.asellow@yahoo.com

\section{Introduction}

Water is essential to all forms of life and makes up 50-97\% of the weight of all plants, animals and about $70 \%$ of the human body. Despite its importance, water is the most poorly managed resource in the world. The main sources of water pollution are domestic sewage, industrial effluent, and agricultural runoff. Sodium, copper, chromium, lead and mercury are some of the more common effluent pollutants discharged from industries which causes a number of hazardous diseases. So there is a very urgent need to control heavy metal emissions into the environment [1-4].

Nowadays filtration, microfiltration and even ultrahigh filtration were performed using polymeric membranes [5]. Natural degradable polymers and their composites are amongst these materials. Many efforts were made to enhance their workability via blending [6] or adding dopant in the nanoscale [7].

Chitosan consider one of the unique renewable and abundant cationic polysaccharide polymeric material results from the deacetylation process of Chitin and characterized their biodegradability and nontoxicity. Chitosan is a functional biopolymer used extensively pharmaceutics, food and in biological applications as immune enhancing partner, anti-tumoral and anti-fungal agent [8-10].

Cellulose is the primary component of the core composition of living plant cell wall and may be considered as the major constituent of plant biomass which are the most abundant biopolymer on the earth. Cellulose acetate was synthesized through reaction of cellulose, acetic acid, and acetic anhydride [7]. 
The most of commercially synthetic artificial membranes in the separation industry are made of polymeric matrices. Which may be a single virgin polymer or mix of two different polymers with various characteristic that result in a new material with unique properties for different applications

Present work aims to synthesis and optimized a novel biodegradable Chitosan/Cellulous Acetate $(\mathrm{Ch} / \mathrm{CA})$ natural polymeric membrane prepared via green route (solvent free) method to be used in water treatment applications.

\section{Experimental Work}

\subsection{Materials}

Commercial grade Cellulose acetate was supplied from winlab co., UK. Commercial grade Chitosan (poly2 -aminoglucose) of deacetylation degree DD $=93 \%$ was supplied by SIGMA Aldrich Co.

Polymeric membrane of varying weight fractions were prepared. Chitosan was dissolved in $2 \%$ aqueous solution of acetic acid while cellulose acetate was dissolved in glacial acetic acid. Prepared solution added to each other drop wise and vigorously stirred at room temperature for about 3 hours. Obtained solution were then poured in plastic petri dishes kept at about $50^{\circ} \mathrm{C}$ until all solvent traces was evaporated. Dry films peeled from the dishes and kept in a vacuum dissector until use. Table 1 shows the chemical composition of prepared thin membrane.

Table (1) chemical composition of prepared thin membrane

\begin{tabular}{|c|c|c|c|c|c|}
\hline ple $^{\text {Sam }}$ & $\mathrm{Ch}^{10}$ & Ch ${ }^{20}$ & Ch ${ }^{30}$ & Ch ${ }^{40}$ & Ch ${ }^{50}$ \\
\hline Ch & 10 & 20 & 30 & 40 & 50 \\
\hline CA & 90 & 80 & 70 & 60 & 50 \\
\hline
\end{tabular}

\subsection{Methods}

Fourier transform infrared (FT-IR) experimental data were collected for three different samples from each composition with 32 scan for each using Nicolet is 10 spectrophotometer in the spectral range $4000-400 \mathrm{~cm}^{-1}$ with a resolution $4 \mathrm{~cm}^{-1}$. X-ray diffraction scans (XRD) obtained using DIANO corporation (USA) equipped with CuK $\alpha$ radiation $(\lambda=1.79026 \hat{A})$, the tube operated at $30 \mathrm{kV}$ within the Bragg angle $(2 \theta)$ ranging between $\left[5-80^{\circ}\right]$. Ultraviolet/visible (UV/Vis.) absorption spectra were measured at the wavelength region of [200-600] $\mathrm{nm}$ using a spectrometer (Perkin-Elmer UV/Vis). SEM micrographs were performed on thin polymeric films of suitable thickness after coating with gold layer of thickness around $2 \mathrm{~nm}$ via JEOL JSM-6510LV, USA, operating at 20 $\mathrm{kV}$ accelerating voltage.

\section{Results and discussion}

\subsection{X-ray diffraction}

Figure (1) reveals X-ray diffraction (XRD) of virgin basic samples (chitosan and cellulose acetate) prepared via casting route without any further treatment. Obtained data approved the semi crystalline nature of both samples with two halos around 15, 20.7 in case of chitosan and for cellulose acetate associated with (101) and (020) planes. These characteristic halos previously described by Sudha et al [11] and Mututuvari et al. [12]. 


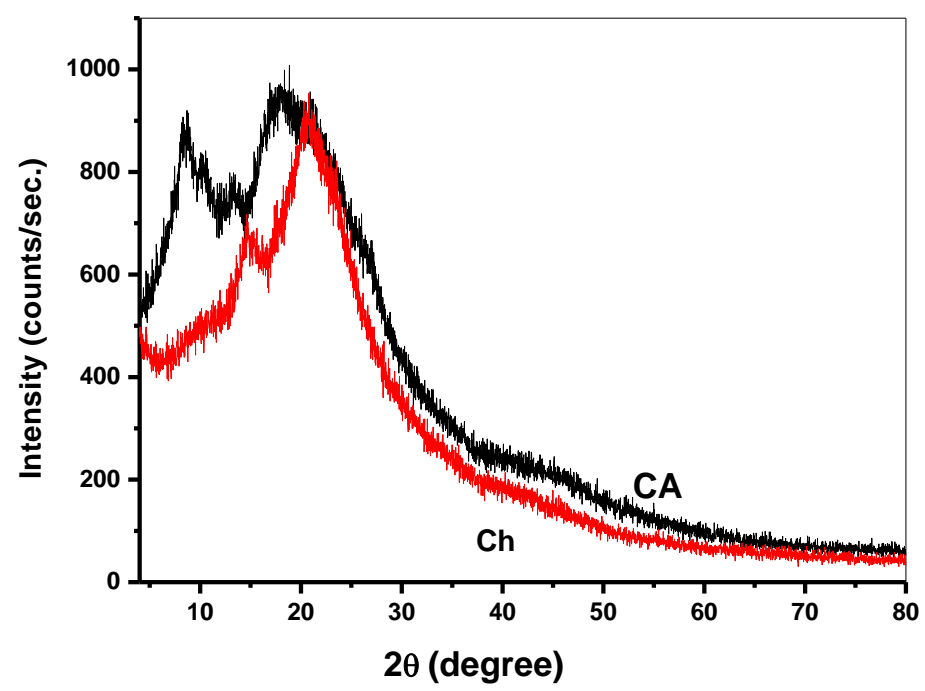

Figure (1): X-ray diffraction scans of pure chitosan and cellulose acetate

Figure (2) shows XRD experimental data of the prepared polymer blend with variable concentration of both samples. First insight for the complex spectra reveal the amorphous character of all prepared samples with obvious increase in amorphousity. Disappearance characteristic crystalline peaks present in virgin constituent is a good indication for complexion and interaction between these polymers and allow homogenous distribution of both polymers their prepared films or membranes with all ratios.

Maxima of XRD peaks was found to be varied with increasing content of chitosan pointing to variation of characteristics and role of each constituent in synthesized samples. Obvious change of the $2 \Theta$ angle observed at maximum intensity in synthesized samples of low chitosan content up to (20\%) followed by gradual decrease tell sample containing about $50 \%$ chitosan.

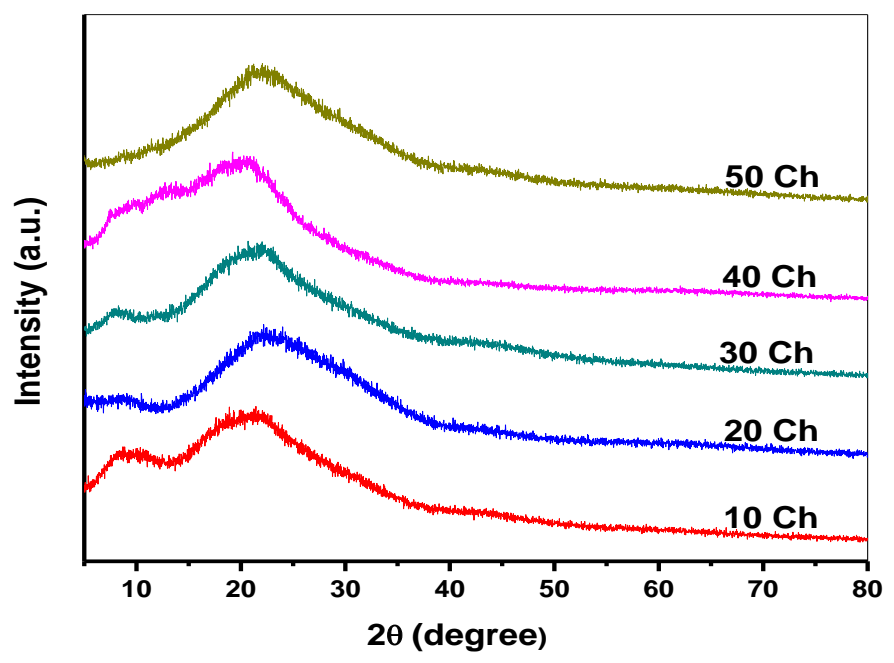

Figure (2): X-ray diffraction scans of Ch/CA blend films.

\subsection{FTIR absorption spectra}

Infrared spectroscopy given a variable non-destructive, rapid and accurate technique that used for the identification of specified functional groups especially in case of organic compounds different route can be 
used in case of inorganic samples for composite containing minor concentration of organic phase. Figure (3) reveals FTIR normalized absorption of synthesized polymeric thin films in combination with their variable concentration blends. FTIR optical absorption spectrum of chitosan sample shows a prominent broad band located at and of measurement at about $3447 \mathrm{~cm}^{-1}$ assigned to the presence of both $\mathrm{OH}$ and $\mathrm{NH}$ stretching variations, a small broad band can be observed at 2927 and $2883 \mathrm{~cm}^{-1}$ assigned to $\mathrm{CH}_{3}$ asymmetric and symmetric stretching vibrations $[8-13,14]$.

The bands located in the region $(2000 \rightarrow 400) \mathrm{cm}^{-1}$ considered as a finger print of nearly natural polymers and contains characteristic bands with variable intensities that depends on the structure related to bond length and/or bond angle. Table (2) list the assignment of different FTIR characteristic bands of both chitosan and cellulose acetate natural polymeric thin films in addition to the different mass fraction blend.

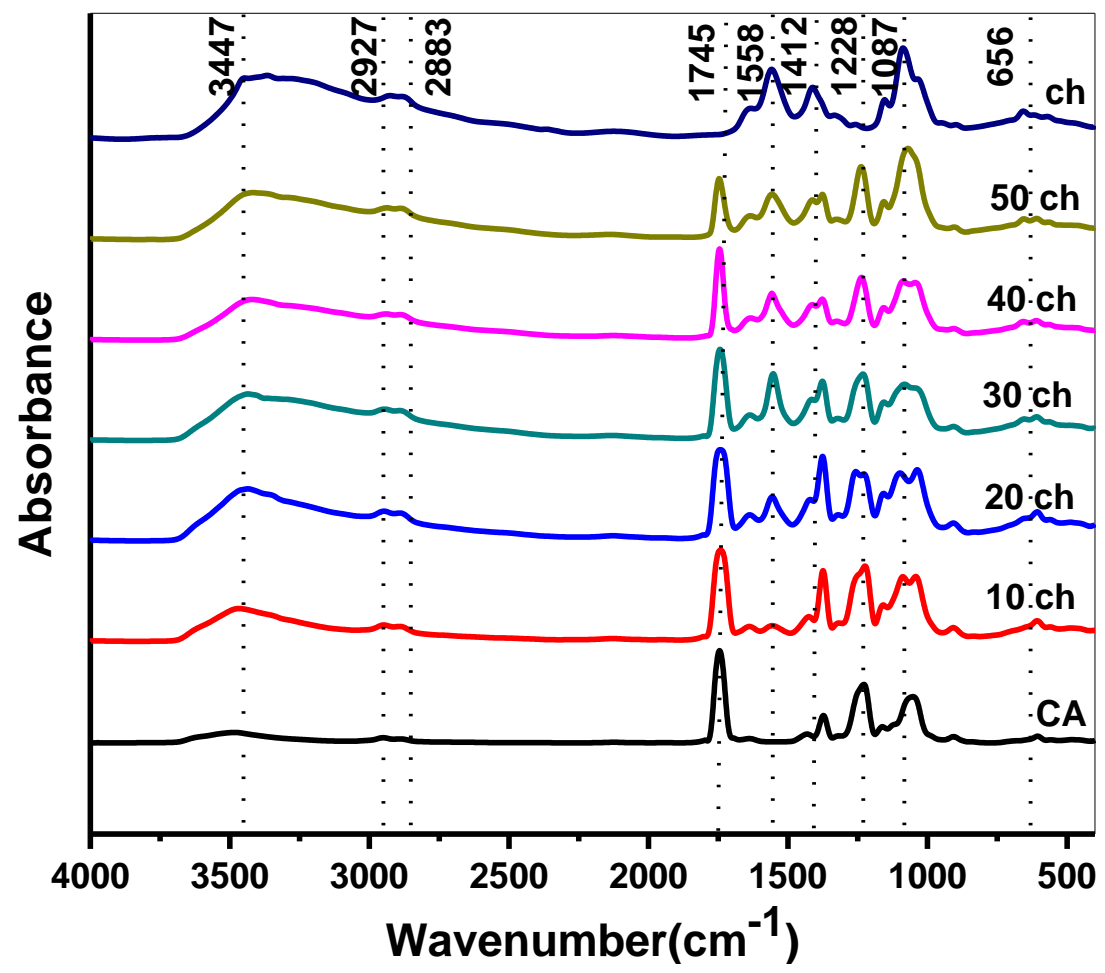

Figure (3): shows FTIR of pristine polymers and blend samples containing variable amount of $\mathrm{Ch} / \mathrm{CA}$.

Table (2): Assignment of the IR characterizing peaks of Chitosan and Cellulose acetate system $[8,12-15]$.

\begin{tabular}{cc}
\hline Assignment & Wavenumber $\left(\mathbf{c m}^{\mathbf{- 1}}\right)$ \\
\hline$(\mathrm{O}-\mathrm{H})$ Stretching & 3447
\end{tabular}

$\left(\mathrm{CH}_{3}\right)$ Asymmetric stretching 2951

$\left(\mathrm{CH}_{3}\right)$ Symmetric stretching $\quad 2890$

$(C=O)$ Stretching $\quad 1745$

$(\mathrm{C}=\mathrm{O})$ bond Stretching $\quad 1639$

$(\mathrm{N}-\mathrm{H})$ Stretching of Amide II 1558

$\left(\mathrm{CH}_{3}\right)$ Symmetric deformation $\quad 1412$ 
$\left(\mathrm{CH}_{3}\right)$ Asymmetric deformation

Amide III Stretching

Acetate (C-C-O) Stretching

1162

(Characteristic to saccharide structure)

898

(C-O) Stretching

1052

\subsection{UV/Visible optical absorption spectra}

Figure (4) displays UV/Vis optical absorption spectral data of all synthesized samples in the spectral range 200-1000 nm. All samples shows the characteristic sharp absorption edge located between $205-245 \mathrm{~nm}$ shifts to higher wavelength with increasing chitosan content. This shift indicate some type of variation in the optical energy gap that can be related to the change in degree of crystallinity or amorphucity in the synthesized sample. The remind spectral range (visible section) shows nearly no change and characterized by the absence of any sharp or broad band. The band located in the UV section may be attributed mainly to unsaturated $\mathrm{C}=\mathrm{O}$ and/or $\mathrm{C}=\mathrm{C}$ bands assigned to $\pi \rightarrow \pi^{*}$ transition within the polymeric matrices. The knick or shoulder appears at about $300-450 \mathrm{~nm}$ in the blend samples arising from $\mathrm{N}$ double bond and may be assigned to $n \rightarrow \pi^{\star}$ transitions within the polymeric matrices $[16,17]$.

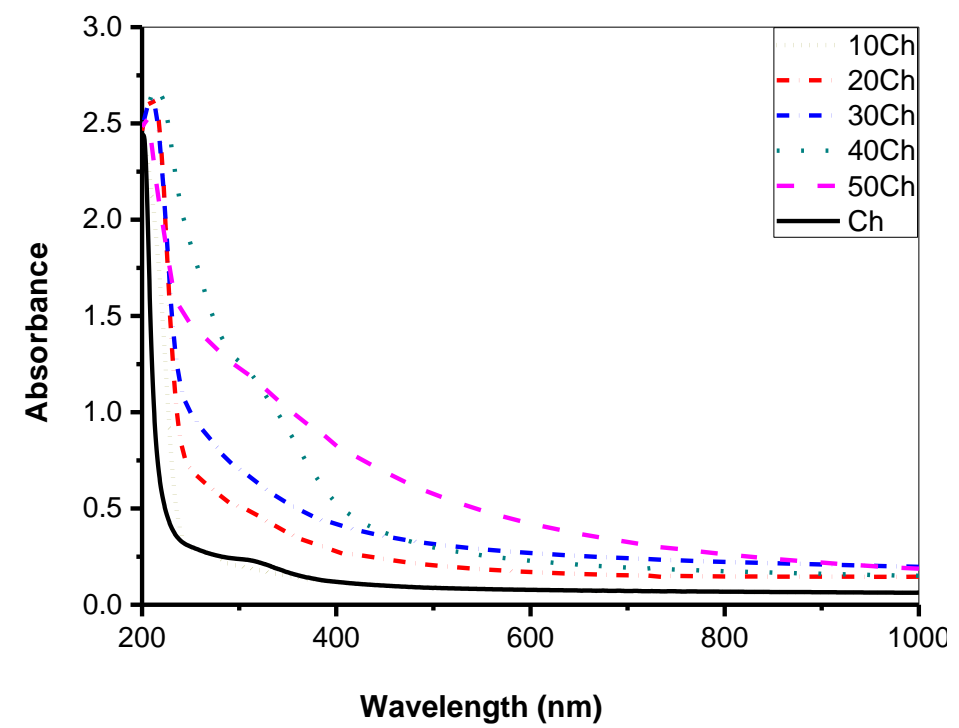

Figure (4): UV-Vis. Spectra of pure chitosan and cellulose acetate films

and their blend by different concentrations.

Further analysis can be performed via calculation of possible transitions and values of the optical energy gap using famous formula that correlate photon energy (hu) with both absorption coefficient and index r.

$$
(\alpha h u)=c(h u-E g)^{r} \quad h u>E g
$$

For direct, indirect allowed and forbidden transitions by plotting the relation between hu versus $(\alpha h v)^{1 / 2}$ and $(\alpha h v)^{2}$ consequently for both direct allowed and indirect allowed transitions as an exemplified in Figure (5). 


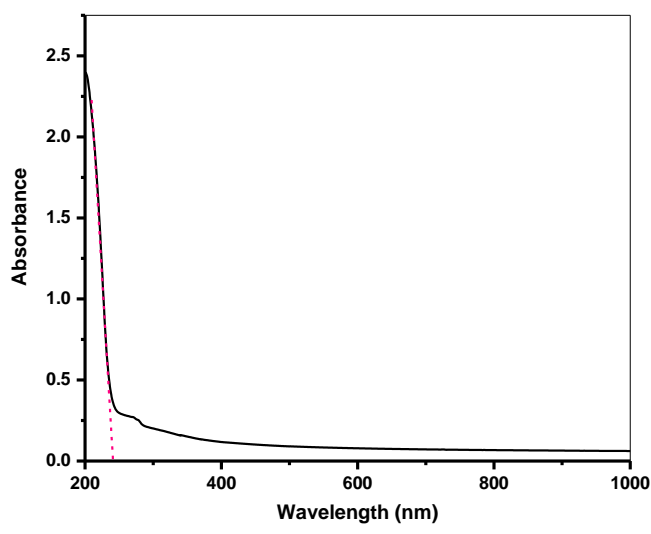

(a)

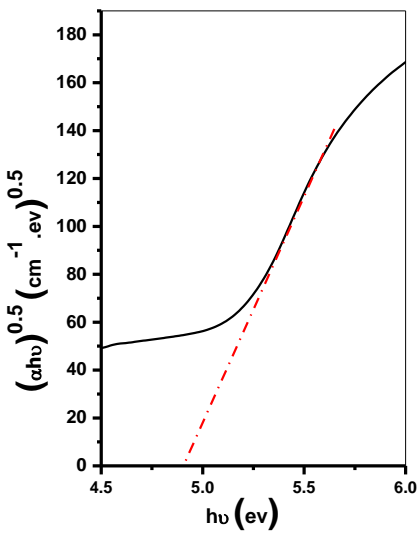

(b)

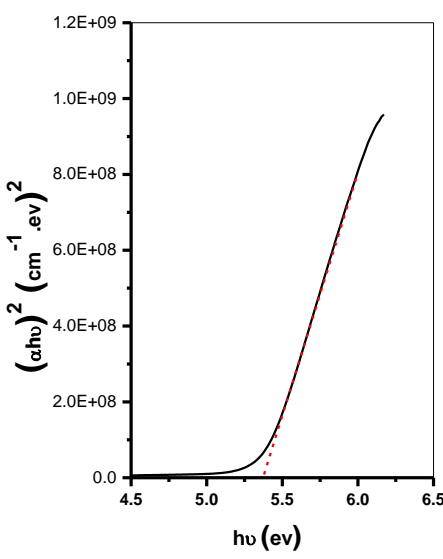

(c)

Figure (5) (a) UV-Vis spectra of exemplified blend sample containing $10 \%$ chitosan, photon energy versus (b)

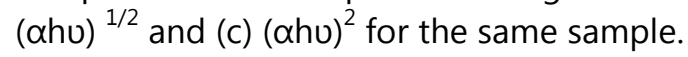

The optical energy gap in electron volts (ev) for all samples were calculated from intersection of extrapolated linear section with $\mathrm{x}$-axis and tabulated in table (3).

Table (3) calculated optical energy gap of synthesized samples

\begin{tabular}{ccccc}
\hline Ch\% & \multicolumn{2}{c}{$\mathbf{E}_{\mathbf{g}}(\mathbf{e V})$} & Absorption edge & $\begin{array}{c}\mathbf{E}_{\mathbf{g}}(\mathbf{e V}) \\
\text { Davis and Mott }\end{array}$ \\
\cline { 2 - 3 } & $\mathbf{E}_{\mathbf{g d}}$ & $\mathbf{E}_{\mathbf{g} \mathbf{i}}$ & $\boldsymbol{\lambda}_{\mathbf{g}}(\mathbf{n m})$ & $\begin{array}{c}\text { Tauc's } \\
\text { expression }\end{array}$ \\
\hline 10 & 4.82 & 5.39 & 224.34 & 5.16 \\
20 & 4.64 & 5.31 & 245.36 & 5.13 \\
30 & 4.41 & 5.24 & 263.86 & 5.02 \\
40 & 3.00 & 4.78 & 277.87 & 4.19 \\
50 & 3.33 & 5.20 & 291.89 & 5.40 \\
\hline
\end{tabular}




\subsection{Scanning electron microscopic analysis (SEM)}

Detailed information about surface morphology and topography associated with variable change in composition and interactions may be retraced using SEM route. Figure (6.a-g) shows the flaky nature of both chitosan and cellulose acetate matrix in different concentrations. Presented images shows the formation of homogenous structure with fair distribution of both polymers by naked eye resulting from the nature of preparation route involving dissolution process in aqueous solution containing $2 \%$ acetic acid in case of chitosan and glacial acetic acid in case of cellulose acetate samples. Expect in the case of higher concentration of both partner (CA/Ch) samples (c, d, e, $f$ and $g)$.

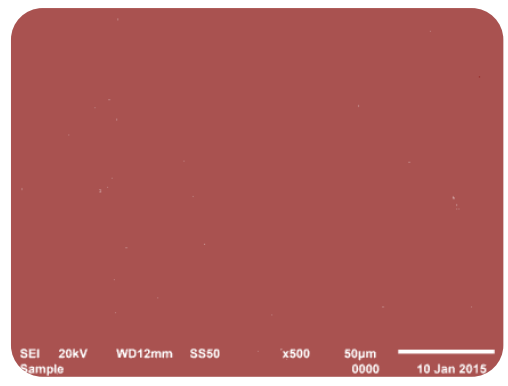

(a)

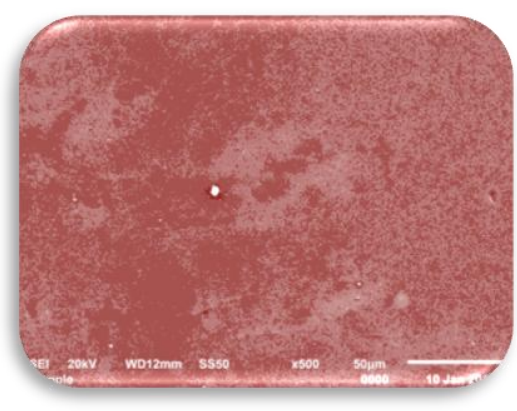

(c)

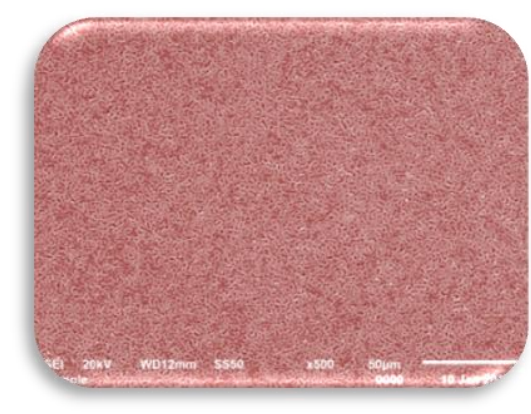

(f)

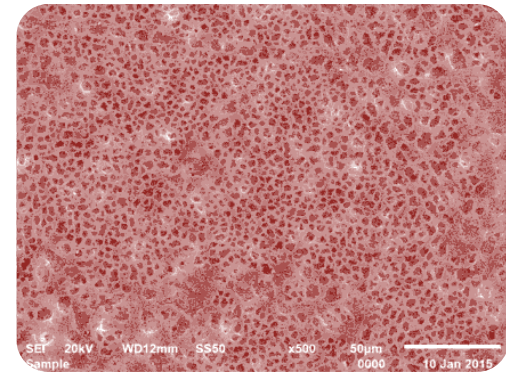

(b)

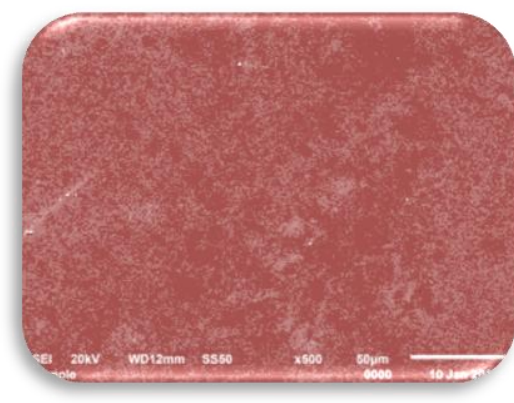

(d)

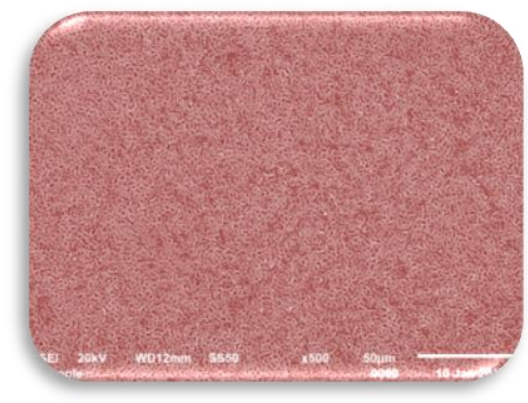

(e)

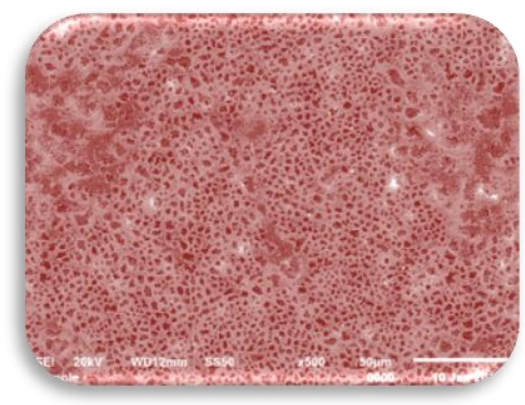

(g)

Figure (6): SEM micrograph of pure chitosan, cellulose acetate films and their variable mass fraction blend (a. Ch, b. CA, c. 10, d. 20, e. 30, f. 40, and g. 50 wt\% Ch) 


\section{Conclusions}

Casting method was applied successfully for preparation of Chitosan (Ch) and Cellulose Acetate (CA) natural polymer membrane in combination with variable mass fraction blend (Ch/CA). X-ray diffraction (XRD) analysis reveals the amorphous nature of all prepared samples. Obvious change in maxima position at low chitosan content up to (20\%) followed by gradual decrease tell sample containing about $50 \%$ chitosan. Observed variation in both position and intensity were observed in FTIR spectral data indicating some type of interaction between constituent polymers and supported by XRD data. UV/vis. spectral absorption curves shows no distinct variation in the visible region combined with appearance of absorbance in the UV section attributed mainly to unsaturated $\mathrm{C}=\mathrm{O}$ and/or $\mathrm{C}=\mathrm{C}$ bands assigned to $\pi \rightarrow \pi^{*}$ transition while the shoulder appears at about 300-450 nm considered to be arising from $N$ double bond which may be assigned to $n \rightarrow \pi^{*}$ transitions within the polymeric matrices. SEM micrographs point out to the formation of homogenous structure with fair distribution of both polymers resulting from the nature of preparation route and involving dissolution process.

\section{References}

1. W. Kaim and B. Chwederski, Bioinorganic Chemistry: Inorganic Elements in the Chemistry of Life, An Introduction and Guide, Wiley, New York, 1994, pp. 151-161.

2. C.G. Fraga. Relevance, essentiality and toxicity of trace elements in human health. Molecular Aspects of Medicine 26 (2005) 235-244.

3. A. Szpanowska-Wohn, E. Kolarzyk, E. Chowaniec, Estimation of intake of zinc, copper and iron in the diet of patients with chronic renal failure treated by haemodialysis. Biol. Trace Elem. Res. 124(2)(2008)97-102.

4. Esfahani ST, Hamidian MR, Madani A, Ataei N, Mohseni $P$, Roudbari $M$, Haddadi M. Serum zinc and copper levels in children with chronic renal failure. Pediatr Nephrol. 21(8)(2006)11531156.

5. A. Ghaee, M. Shariaty-Niassar, J. Barzin, T. Matsuura, Effects of chitosan membrane morphology on copper ion adsorption, Chem. Eng. J. 165 (2010) 46-55.

6. R.S. Vieira, E. Guibal, E.A. Silva, M.M. Beppu, Adsorption and desorption of binary mixtures of copper and mercury ions on natural and crosslinked chitosan membranes, Adsorption 13 (2007) 603-611.

7. C. Liu, R. Bai, Adsorptive removal of copper ions with highly porous chitosan/ cellulose acetate blend hollow fiber membranes, J. Membr. Sci. 284 (2006) 313-322.

8. Z. Cheng, X. Liu, M. Han, W. Ma, Adsorption kinetic character of copper ions onto a modified chitosan transparent thin membrane from aqueous solution, J. Hazard. Mater. 182 (2010) 408-415.

9. R.M. Nthumbi, J.C. Ngila, A. Kindness, B. Moodley, L. Petrik, Method Development for Flow Adsorption and Removal of Lead and Copper in Contaminated Water Using Electrospun Nanofibers of Chitosan Blend, Analytical Letters, 44 (2011) 1937-1955.

10. B. Gajda, M.B. Bogacki, The application of polymer inclusive membranes for removal of heavy metal ions from waste solutions, Journal of Achievements in Materials and Manufacturing Engineering 55(2)(2012)673-678.

11. P.N. Sudha, P.A. Vinodhini, K. Sangeetha, S. Latha, T. Gomathi, Fabrication of cellulose acetatechitosan-polyethylene glycol ultrafiltration membrane for chromium removal, Der Pharmacia Lettre, 6 (1)(2014)37-46. 
12. T.D. Kusworo, J. Supriyadi and D.C. Hakika, Enhanced Separation Performance of Cellulose Acetate Membrane For Brackish Water Separation Using Modification of Additives and Thermal Annealing, Int. J. Waste Resources 4(2014)1.

13. Y.G. Abou El-Reash, A.M. Abdelghany, A. Abd Elrazak, Removal and Separation of $\mathrm{Cu}(\mathrm{II})$ from Aqueous Solutions Using Nano-Silver Chitosan/Polyacrylamide Membranes, International Journal of Biological Macromolecules, 86(2016)789-798.

14. Y.G. Abou El-Reash, A.M. Abdelghany, K. Lepold, Solid phase extraction of $\mathrm{Cu} 2+$ and $\mathrm{Pb}^{2+}$ from waters using new thermally treated Chitosan/ Polyacrylamide thin films; Adsorption kinetics and thermodynamics, International Journal of Environmental Analytical Chemistry, DOI: 10.1080/03067319.2017.1376905.

15. E.M. Abdelrazek, I.S. Elashmawi, S. Labeeb, Chitosan filler effects on the experimental characterization, spectroscopic investigation and thermal studies of PVA, Physica B 405(2010)2021-2027

16. E.M. Abdelrazek, A.M. Abdelghany, S.I. Badr, M.A. Morsi, Evaluation of optical parameters and structural variations of UV irradiated (PEO/PVP)/Au polymer nanocomposites, Research Journal of Pharmaceutical Biological and Chemical, 7(2) (2016)1877-1890.

17. A.M. Abdelghany, M.A. Morsi, A. Abdelrazek, M.T. Ahmed, Role of silica nanoparticles on structural, optical and morphological properties of Poly(Vinyl Chloride-co-Vinyl Acetate-co-2- Hydroxypropyl Acrylate) copolymer, Silicon, 10.1007/s12633-016-9483-z. 